\title{
HAUSDORFF DIMENSION IN GRAPH DIRECTED CONSTRUCTIONS
}

\author{
R. DANIEL MAULDIN AND S. C. WILLIAMS
}

\begin{abstract}
We introduce the notion of geometric constructions in $\mathbf{R}^{m}$ governed by a directed graph $G$ and by similarity ratios which are labelled with the edges of this graph. For each such construction, we calculate a number $\alpha$ which is the Hausdorff dimension of the object constructed from a realization of the construction. The measure of the object with respect to $\mathscr{l}^{\alpha}$ is always positive and $\sigma$-finite. Whether the $\mathscr{H}^{\alpha}$-measure of the object is finite depends on the order structure of the strongly connected components of $G$. Some applications are given.
\end{abstract}

A geometric graph directed construction in $\mathbf{R}^{m}$ consists of

(1) a finite sequence of nonoverlapping, compact subsets of $\mathbf{R}^{m}: J_{1}, \ldots, J_{n}$ such that each $J_{i}$ has a nonempty interior,

(2) a directed graph $G$ with vertex set consisting of the integers $1, \ldots, n$ and similarity maps $T_{i, j}$ of $\mathbf{R}^{m}$, where $(i, j) \in G$, with similarity ratios $t_{i, j}$ such that

(a) for each $i, 1 \leq i \leq n$, there is some $j$ such that $(i, j) \in G$,

(b) for each $i,\left\{T_{i, j}\left(J_{j}\right) \mid(i, j) \in G\right\}$ is a nonoverlapping family and

$$
J_{i} \supseteq \bigcup\left\{T_{i, j}\left(J_{j}\right) \mid(i, j) \in G\right\}
$$

and

(c) if the path component of $G$ rooted at the vertex $i_{1}$ is a cycle:

$$
\left[i_{1}, \ldots, i_{q}, i_{q+1}=i_{1}\right]
$$

then

$$
\prod_{k=1}^{q} t_{i_{k}, i_{k+1}}<1
$$

We will generalize the setting to ratio graph directed constructions later in the paper.

Each geometric construction naturally determines a compact subset $K$ of $\mathbf{R}^{m}$. This set, which we will term the construction object, is pieced together by following the graph $G$ and applying the maps coded by the edges to the corresponding sets. To make explicit how this is done we will first formulate how the pieces are generated. For each $i$, let $\mathscr{K}\left(J_{i}\right)$ be the space of compact subsets of $J_{i}$ provided with the Hausdorff metric, $\rho_{H}$.

Received by the editors August 17, 1987.

1980 Mathematics Subject Classification (1985 Revision). Primary 28C10.

Key words and phrases. Spectral radius, entropy, Hausdroff measure.

The research of the first author was supported in part by a grant from the National Science Foundation. 
THEOREM 1. For each goemetric construction, there exists a unique vector of compact sets, $\left(K_{1}, \ldots, K_{n}\right) \in \prod_{i=1}^{n} \mathscr{K}\left(J_{i}\right)$ such that for each $i$,

$$
K_{i}=\bigcup\left\{T_{i, j}\left(K_{j}\right) \mid(i, j) \in G\right\}
$$

The construction object is defined as

$$
K=\bigcup_{i=1}^{n} K_{i}
$$

As the proofs of our theorems show, it is really only the regular part of $J_{i}$ that counts. Thus, we could assume each $J_{i}=\operatorname{cl}\left(\right.$ int $\left.J_{i}\right)$ to begin with. Our main results concern the Hausdorff dimension $\alpha$ of the set $K$ and the measure of $K$ with respect to the corresponding Hausdorff measure $\mathscr{H}^{\alpha}$. In order to formulate these results, we must present another fundamental tool associated with a construction and set some terminology.

The weighted incidence matrix or construction matrix $A=A_{G}$ associated with a graph directed construction is the $n \times n$ matrix defined by

$$
A=\left[t_{i, j}\right]_{i, j \leq n},
$$

where we make the convention that $t_{i, j}=0$ if $(i, j) \notin G$. For each $\beta \geq 0$, let $A_{\beta}=A_{G, \beta}$ be the $n \times n$ matrix given by $a_{\beta ; i, j}=t_{i, j}^{\beta}$. Also, let $\Phi(\beta)$ be the spectral radius of $A_{\beta}$. Of course, according to the Frobenius-Perron theorem, $\Phi(\beta)$ is the largest nonnegative eigenvalue of $A_{\beta}$. It is known that $\Phi$ is continuous.

THEOREM 2. $\Phi(0) \geq 1, \Phi$ is continuous, strictly decreasing, and $\lim _{\beta \rightarrow \infty} \Phi(\beta)$ $=0$. Indeed, there is a number $c, 0<c<1$, such that for all $\beta \geq 0$ and $\varepsilon>0$, $\Phi(\beta+\varepsilon) \leq c^{\varepsilon} \Phi(\beta)$.

For each graph directed construction, let $\alpha$ be the nonnegative number such that $\Phi(\alpha)=1$. We will show that the dimension of the construction object $K$ is $\alpha$. This will be done by showing that $K$ has positive $\mathscr{H}^{\alpha}$ measure and, moreover, the measure of $K$ is either finite or $\sigma$-finite depending on the structure of the graph $G$. We set some terminology concerning graphs.

A cycle is a directed graph $H$ for which there is a closed path which passes into every vertex exactly once and such that every edge of $H$ is an edge of this path. A directed graph $H$ is said to be strongly connected provided that whenever each of $x$ and $y$ is a vertex of $H$, then there is a directed path from $x$ to $y$.

A strongly connected component of $G$ is a maximal subgraph $H$ of $G$ such that $H$ is strongly connected. Of course, the strongly connected components of $G$ are pairwise disjoint. It is possible that they do not cover $G$. It is also possible for such a component to consist of a single vertex looped on itself. A vertex is not considered to be strongly connected unless it is looped on itself.

REMARK. If $G$ is a construction graph, then $G$ must have at least one strongly connected component. 
A classical theorem states that determinants may be factored over the strongly connected components. In our case, we have

FACTOR THEOREM. If $H_{1}, H_{2}, \ldots, H_{s}$ are the strongly connected components of $G$, then

$$
\operatorname{det}\left(I-A_{G, \beta}\right)=\prod_{i=1}^{s} \operatorname{det}\left(I-A_{H_{i}, \beta}\right) .
$$

The path component of $G$ rooted at a vertex $i$ consists of all vertices $j$ such that there is a directed path from $i$ to $j$. To say that a path component is a cycle means that the subgraph of $G$ over that component is a cycle. Clearly, if a path component is a cycle $H$, then $H$ is a strongly connected component of $G$. For each subgraph $H$ of $G$, let $V(H)$ be the vertex set of $H$. However, we will normally write $i \in H$ instead of $i \in V(H)$.

We give a sufficient condition for $\mathscr{H}^{\alpha}(K)$ to be finite. The development of Hausdorff measures may be found in Falconer [6] or Rogers [8].

THEOREM 3. For each graph directed construction such that $G$ itself is strongly connected, the Hausdorff dimension of $K$, the construction object, is $\alpha$, where $\Phi(\alpha)=1$. Moreover,

$$
0<\mathscr{H}^{\alpha}(K)<+\infty .
$$

In order to analyze a construction, we get the following notation. Set $G(1)=$ $\{1, \ldots, n\}$ and for each integer $p \geq 2$, set

$$
G(p)=\left\{\left(i_{1}, \ldots, i_{p}\right) \in\{1, \ldots, n\}^{p} \mid\left(i_{j}, i_{j+1}\right) \in G, j=1, \ldots, p-1\right\} .
$$

Also, set

$$
G^{*}=\bigcup_{p=1}^{\infty} G(p)
$$

and

$$
G^{\infty}=\left\{\left(i_{j}\right) \in\{1, \ldots, n\}^{\mathbf{N}} \mid\left(i_{j}, i_{j+1}\right) \in G, j=1,2,3, \ldots\right\} .
$$

We use the partial order on $G^{*} \cup G^{\infty}$ given by $\sigma<\tau$ provided $\tau$ extends $\sigma$.

At this point let us mention that there is some overlap of our Theorem 3 and some results of $\mathrm{T}$. Bedford [11]. Bedford considers the case where the maps $T_{i j}$ depend only on $j$ and the sets $J_{j}$ are convex. It is not determined whether the $\mathscr{H}^{\alpha}$ measure is finite or $\sigma$-finite, but rather that it is equivalent to the Gibbs measure.

We turn now to the interesting case when $A$ is not assumed to be irreducible. If the construction matrix $A$ is not irreducible, then the measure of $K$ depends on the order structure of the strongly connected components of $G$. Let $\operatorname{SC}(G)$ be the set of all strongly connected components of $G$. Each $H \in \operatorname{SC}(G)$ defines a graph directed subconstruction. This subconstruction, based upon the sets $J_{i}$ such that $i \in V(H)$, has directed graph $H$, and the similarity maps are those from the original construction. Clearly, a subconstruction satisfies conditions 2(a) and 2(b). We will verify later that condition $2(\mathrm{c})$ is also satisfied. For each $H \in \mathrm{SC}(G)$, let $\alpha_{H}$ be the number $\beta$ such that $\Phi_{H}(\beta)=1$. We partially order $\operatorname{SC}(G)$ by stating that $H_{1} \preceq H_{2}$ provided there is a path $\gamma=\left\{g_{1}, \ldots, g_{k}\right\} \in G^{*}$ such that $g_{1} \in H_{1}$ and $g_{k} \in H_{2}$.

Our most general result presented here can now be stated. 
THEOREM 4. For each graph directed construction, $\alpha=\max \left\{\alpha_{H} \mid H \in \mathrm{SC}(G)\right\}$ and $K$ has positive $\sigma$-finite $\mathscr{H}^{\alpha}$ measure. Further, $\mathscr{H}^{\alpha}(K)<\infty$ if and only if $\left\{H \in \mathrm{SC}(G) \mid \alpha_{H}=\alpha\right\}$ consists of pairwise incomparable elements.

For each $\sigma=\left(i_{1}, \ldots, i_{p}\right) \in G^{*}$ with $|\sigma|=p \geq 2$, set

$$
\begin{gathered}
T_{\sigma}=T_{\sigma(1), \sigma(2)} \circ \cdots \circ T_{\sigma(p-1), \sigma(p)}, \\
t_{\sigma}=\prod_{i=1}^{p-1} t_{\sigma(i), \sigma(i+1)},
\end{gathered}
$$

and,

$$
J_{\sigma}=T_{\sigma}\left(J_{\sigma(p)}\right) .
$$

These sets form a Cantor scheme. Thus, for $p=1,2,3, \ldots$ the sets $\left\{J_{\sigma} \mid \sigma \in G(p)\right\}$ are nonoverlapping and if $\sigma$ and $\tau$ are in $G^{*}$ and $\sigma<\tau$, then $J_{\tau} \subset J_{\sigma}$. In addition, for each $\sigma$ in $G(p), \operatorname{int}\left(J_{\sigma}\right) \neq \varnothing$ and

$$
\operatorname{diam} J_{\sigma}=t_{\sigma} \operatorname{diam} J_{\sigma(|\sigma|)} .
$$

Note that the construction object $K$ can be expressed as

$$
K=\bigcap_{p=1}^{\infty}\left[\bigcup_{\sigma \in G(p)} J_{\sigma}\right] .
$$

Our analysis of the dimension and measure of $K$ depends upon a detailed study of the natural approximating sums determined by the $n$th level sets $J_{\sigma}$ in (15).

LEMMA I. If $\tau=\left[i_{1}, i_{2}, \ldots, i_{l}, i_{l+1}=i_{1}\right]$ is a cycle in $G$, then

$$
t_{\tau}<1 .
$$

ProOF. Let $H$ be the strongly connected component of $G$ with $\tau \subseteq H$. If $\tau$ is the path component of $i_{1}$, then by condition 2(c), (16) holds.

Otherwise, $\tau$ is a proper subset of $H$ and for some vertex $i_{s}$ of $\tau$ and some $j \in H \backslash \tau$, there is an arrow from $i_{s}$ to $j$.

Consider $J_{\left(i_{1}, \ldots, i_{s}, i_{s+1}\right)}$ and $J_{\left(i_{1}, \ldots, i_{s}, j\right)}$. Since these sets are nonoverlapping nonempty subsets of $J_{i_{1}}$ with nonempty interiors, $J_{i_{1}, \ldots, i_{l}, i_{1}}=T_{i_{1}, i_{2}} \circ \cdots \circ T_{i_{l}, i_{1}}\left(J_{i_{1}}\right)$ is a proper subset of $J_{i_{1}}$. Therefore, $t_{\tau}<1$, as was to be proved.

For each cycle $\tau$ in $G$, let $\# \tau$ be the number of vertices in the subgraph $\tau$. Set

$$
\Gamma=\sup \left\{\left(t_{\tau}\right)^{1 / \# \tau} \mid \tau \text { is a cycle }\right\} .
$$

LEMMA II.

$$
\lim _{p \rightarrow \infty}\left[\sup _{\sigma \in G(p)} t_{\sigma}\right]^{1 / p} \leq \Gamma<1 .
$$

Proof. Fix $\sigma=\left(i_{1}, \ldots, i_{p}\right) \in G(p)$, with $p>n$. By the pigeon-hole principle some index is repeated. Choose a cycle in $\sigma$, factor the product over the cycle from the entire product, and remove the cycle from $\sigma$ except for one of the repeated 
ındices. We again obtain a path. Continue. Thus, for each cycle $\zeta$, there is a nonnegative integer $q_{5}$ such that

$$
t_{\sigma}=\prod_{\varsigma} t_{\varsigma}^{q_{\varsigma}} t_{\tau}
$$

where $\tau \in G^{*}$ and $|\tau| \leq n$. Thus,

$$
t_{\sigma}=\prod_{\varsigma}\left[\left(t_{\varsigma}\right)^{1 / \# s}\right]^{(\# \varsigma) q_{s}} t_{\tau} \leq \Gamma^{\Sigma(\# s) q_{s}} t_{\tau} .
$$

Taking the $p$-root of both sides and then letting $p$ go to infinity, we have (18).

PROOF OF THEOREM 1. Define the map $\Psi$ of $\prod_{i=1}^{n} \mathscr{K}\left(J_{i}\right)$ into itself by

$$
\Psi\left(M_{1}, \ldots, M_{n}\right)=\left(N_{1}, \ldots, N_{n}\right),
$$

where

$$
N_{i}=\bigcup\left\{T_{i, j}\left(M_{j}\right) \mid(i, j) \in G\right\} .
$$

By recursion, the $p$ th iterate of $\Psi$ satisfies

$$
\Psi^{p}\left(M_{1}, \ldots, M_{n}\right)=\left(\Psi_{i}^{p}\left(M_{1}, \ldots, M_{n}\right)\right)_{i=1}^{n},
$$

where

$$
\Psi_{i}^{p}\left(M_{1}, \ldots, M_{n}\right)=\bigcup_{\substack{\sigma \in G(p+1) \\ \sigma(1)=i}} T_{\sigma}\left(M_{\sigma(p+1)}\right) .
$$

Consider the space $\prod_{i=1}^{n} \mathscr{K}\left(J_{i}\right)$ provided with the distance $d$ given by the maximum of the Hausdorff distance between corresponding coordinates. Thus,

$$
\begin{aligned}
& d\left(\Psi^{p}\left(A_{1}, \ldots, A_{n}\right), \Psi^{p}\left(B_{1}, \ldots, B_{n}\right)\right) \\
& \quad=\max _{i \leq n} \rho_{H}\left(\bigcup_{\substack{\sigma \in G(p+1) \\
\sigma(1)=i}} T_{\sigma}\left(A_{\sigma(p+1)}\right), \bigcup_{\substack{\sigma \in G(p+1) \\
\sigma(1)=i}} T_{\sigma}\left(B_{\sigma(p+1)}\right)\right) \\
& \quad \leq \max _{i \leq n} \max _{\sigma \in G(p+1)} t_{\sigma} \rho_{H}\left(A_{\sigma(p+1)}, B_{\sigma(p+1)}\right) \\
& \quad \leq d\left(\left(A_{1}, \ldots, A_{n}\right),\left(B_{1}, \ldots, B_{n}\right)\right) \max _{\sigma \in G(p+1)} t_{\sigma} .
\end{aligned}
$$

By Lemma II, if $p$ is large, $\Psi^{p}$ is a contraction map of $\prod_{i=1}^{n} \mathscr{K}\left(J_{i}\right)$. Thus, $\Psi^{p}$ and therefore, $\Psi$ has a unique fixed point.

NOTE. Let us indicate the relationships between our results and those of Hutchinson [2] (in $\mathbf{R}^{m}$ ) and Moran [1]. Their results may be obtained as follows. Assume $G$ is complete and $J_{1}, \ldots, J_{n}$ are pairwise similar. For each $i$, let $T_{i}$ be a contraction with similarity ratio $r_{i}<1$. Set $T_{i, j}=T_{i}$. Now, from Theorem 1 we find the vector of sets $K_{1}, \ldots, K_{n}$. Setting $K=\bigcup_{i=1}^{n} K_{i}$, we have

$$
K_{i}=T_{i}(K) \text {. }
$$


Therefore, this is Hutchinson's set in this case. Also, in the analogous ratio setting which is given at the end of this paper, we have

$$
A_{\beta}=\left[\begin{array}{cccc}
r_{1}^{\beta} & r_{1}^{\beta} & \ldots & r_{1}^{\beta} \\
\vdots & & & \\
r_{n}^{\beta} & r_{n}^{\beta} & \ldots & r_{n}^{\beta}
\end{array}\right] .
$$

Since all the columns are identical, $\Phi(\beta)=\sum_{i=1}^{n} r_{i}^{\beta}$ and the dimension $\alpha$ of $K$ satisfies

$$
\sum_{i=1}^{n} r_{i}^{\alpha}=1
$$

This is Moran's fundamental formula.

In order to prove Theorem 2 , we recall that if $u=(1, \ldots, 1)$ in $\mathbf{R}^{n}$, then

$$
\lim _{p \rightarrow \infty}\left\|A_{\beta}^{p} u\right\|_{1}^{1 / p}=\Phi_{\beta} .
$$

By recursion, we have, for $p=1,2,3, \ldots$,

$$
\left\|A_{\beta}^{p} u\right\|_{1}=\sum_{\sigma \in G(p+1)} t_{\sigma}^{\beta} .
$$

Let $\Omega=\{1, \ldots, n\}^{\mathbf{Z}}$ and let $S$ be the shift operator on $\Omega$. Let $M$ be the set of all shift-invariant probability measures on $\Omega$ with the weak topology and define $\pi: \Omega \rightarrow\{1, \ldots, n\}^{2}$ by $\pi(\omega)=(\omega(1), \omega(2))$. Let $\Lambda$ be the standard Haar measure on $\Omega$. Thus,

$$
\begin{aligned}
\left\|A_{\beta}^{p} u\right\|_{1} & =n^{p+1} \sum_{\sigma \in G(p+1)}\left(1 / n^{p+1}\right) t_{\sigma}^{\beta} \\
& =n^{p+1} \int_{\Omega} \prod_{j=0}^{p-1} t_{\pi\left(S^{j}(\omega)\right)}^{\beta} d \Lambda(\omega) .
\end{aligned}
$$

Also, let $h(\mu)$ denote the entropy of an element of $M[\mathbf{4}, \mathbf{5}]$.

LEMMA III.

$$
\log \Phi(\beta)=\sup _{\mu \in M}\left\{\beta \int_{\Omega} \log t_{\pi(\omega)} d \mu(\omega)+h(\mu)\right\} .
$$

ProOF. For each $\varepsilon>0$, let $A_{\beta, \varepsilon}$ be the $n \times n$ matrix with entries

$$
t_{\varepsilon,(i, j)}^{\beta}=t_{i, j}^{\beta}+\varepsilon \cdot 1_{G^{\prime}}(i, j)
$$

where $G^{\prime}=\{1, \ldots, n\}^{2} \backslash G$.

Let $\lambda_{\beta, \varepsilon}$ be the largest nonnegative eigenvalue of $A_{\beta, \varepsilon}$. We have $\lim _{\varepsilon \rightarrow 0} \lambda_{\beta, \varepsilon}=$ $\lambda_{\beta}=\Phi(\beta)$ and

$$
\log \lambda_{\beta, \varepsilon}=\lim _{p \rightarrow \infty} \frac{1}{p} \log \left\|A_{\beta, \varepsilon}^{p} u\right\|_{1}
$$


Now,

$$
\begin{aligned}
\left\|A_{\beta, \varepsilon}^{p} u\right\|_{1} & =n^{p+1} \int_{\Omega} \prod_{j=0}^{p-1} t_{\varepsilon, \pi\left(S^{j}(\omega)\right)}^{\beta} d \Lambda(\omega) \\
& =n^{p+1} \int_{\Omega} \exp \left[p\left\{\frac{\beta}{p} \sum_{j=0}^{p-1} \log t_{\varepsilon, \pi\left(S^{j}(\omega)\right)}\right\}\right] d \Lambda(\omega) .
\end{aligned}
$$

For each $\omega=\left(w_{i}\right)_{i=-\infty}^{\infty} \in \Omega$ and positive integer $p$, set

$$
\bar{\omega}_{p}=\left(\ldots, w_{1}, \ldots, w_{p}, w_{1}, \ldots, w_{p}, \ldots\right)
$$

and define a probability measure $R_{p, \omega}$ on $\Omega$ by

$$
R_{p, \omega}=\frac{1}{p} \sum_{j=0}^{p-1} \delta_{S^{j}\left(\bar{\omega}_{p}\right)}
$$

where $\delta_{\eta}$ is the unit mass at $\eta$. Clearly, $R_{p, \omega}$ is shift invariant. Note

$$
\begin{gathered}
\int_{\Omega} \log t_{\varepsilon, \pi(\eta)} d R_{p, \omega}(\eta)-\frac{1}{p} \sum_{j=0}^{p-1} \log t_{\varepsilon, \pi\left(S^{j}(\omega)\right)} \\
=\frac{1}{p}\left(\log t_{\varepsilon,\left(w_{p}, w_{1}\right)}-\log t_{\varepsilon, \pi\left(S^{p-1}(\omega)\right)}\right) .
\end{gathered}
$$

So,

$$
\begin{aligned}
\left\|A_{\beta, \varepsilon}^{p} u\right\|_{1}=n^{p+1} \int_{\Omega}\left[\exp p\left\{\beta \int_{\Omega} \log t_{\varepsilon, \pi(\eta)} d R_{p, \omega}(\eta)\right\}\right] \\
\cdot\left(t_{\varepsilon, \pi\left(S^{p-1}(\omega)\right)} / t_{\varepsilon,\left(w_{p}, w_{1}\right)}\right)^{\beta} d \Lambda(\omega) .
\end{aligned}
$$

Let $M_{\varepsilon}=\max \left\{t_{\varepsilon,(i, j)}\right\}$ and $m_{\varepsilon}=\min \left\{t_{\varepsilon,(i, j)}\right\}$. Thus,

$$
\left\|A_{\beta, \varepsilon}^{p} u\right\|_{1} \leq n^{p+1}\left(M_{\varepsilon} / m_{\varepsilon}\right)^{\beta} \int_{\Omega} \exp \left[p \beta \int_{\Omega} \log t_{\varepsilon, \pi(\eta)} d R_{p, \omega}(\eta)\right] d \Lambda(\omega) .
$$

Therefore,

$$
\begin{aligned}
\lim _{p \rightarrow \infty} \frac{1}{p} & \log \left\|A_{\beta, \varepsilon}^{p} u\right\|_{1} \\
& \leq \log n+\lim _{p \rightarrow \infty} \frac{1}{p} \log \left\{\int_{\Omega} \exp \left[p \beta \int_{\Omega} \log t_{\varepsilon, \pi(\eta)} d R_{p, \omega}(\eta)\right] d \Lambda(\omega)\right\} .
\end{aligned}
$$

We wish to apply Varadhan's theorem [3, Theorem II.7.1]. For each $p$, let $a_{p}=p$ and let $Q_{p}$ be the probability measure defined on the Borel subsets $A$ of $M$ by

$$
Q_{p}(A)=\Lambda\left(\left\{\omega \mid R_{p, \omega} \in A\right\}\right) .
$$

Thus, $Q_{p}$ is the image measure of $\Lambda$ under the Borel measurable map $\omega \rightarrow R_{p, \omega}$ and by the ergodic theorem, $\left\{Q_{p}\right\}_{p=1}^{\infty}$ converges to $\delta_{\Lambda}$. It is shown in [3, Theorem II.4.4(a)] that $\left\{Q_{p}\right\}_{p=1}^{\infty}$ has a large deviation property with constants $\left\{a_{p}\right\}$ and entropy function $I(\mu)=\log n-h(\mu)$. That $I(\mu)$ has this form is shown in $[3$, Ex. I.6.2]. 
Now, according to Varadhan's theorem, we have the variational principle:

$$
\begin{aligned}
\lim _{p \rightarrow \infty} & \frac{1}{p} \log \left\{\int_{M} \exp \left[p \beta \int_{\Omega} \log t_{\varepsilon, \pi(\eta)} d \mu(\eta)\right] d Q_{p}(\mu)\right\} \\
= & \sup _{\mu \in M}\left\{\beta \int_{\Omega} \log t_{\varepsilon, \pi(\omega)} d \mu(\omega)+h(\mu)\right\}-\log n .
\end{aligned}
$$

By changing variables,

$$
\begin{aligned}
\int_{M} \exp \left[p \beta \int_{\Omega} \log t_{\varepsilon, \pi(\eta)} d \mu(\eta)\right] d Q_{p}(\mu) \\
=\int_{\Omega} \exp \left[p \beta \int_{\Omega} \log t_{\varepsilon, \pi(\eta)} d R_{p, \omega}(\eta)\right] d \Lambda(\omega) .
\end{aligned}
$$

From (41), we find

$$
\log \lambda_{\beta, \varepsilon} \leq \sup _{\mu \in M}\left\{\beta \int_{\Omega} \log t_{\varepsilon, \pi(\omega)} d \mu(\omega)+h(\mu)\right\} .
$$

From (39), we also have

$$
\left\|A_{\beta, \varepsilon}^{p} u\right\|_{1} \geq n^{p}\left(m_{\varepsilon} / M_{\varepsilon}\right)^{\beta} \int_{\Omega} \exp \left[p \beta \int_{\Omega} \log t_{\varepsilon, \pi(\eta)} d R_{p, \omega}(\eta)\right] d \Lambda(\omega) .
$$

Proceeding as before, we obtain the reverse inequality in (45). So,

$$
\log \lambda_{\beta, \varepsilon}=\sup _{\mu \in M}\left\{\beta \int_{\Omega} \log t_{\varepsilon, \pi(\omega)} d \mu(\omega)+h(\mu)\right\} .
$$

REMARK. We could have used the notion of topological pressure [4] to obtain (47).

Since

(49) $\log \lambda_{\beta, \varepsilon}=\sup _{\mu \in M}\left\{\beta \int_{\pi(\omega) \in G} \log t_{\pi(\omega)} d \mu(\omega)+\beta \log \varepsilon \mu(\pi(\omega) \notin G)+h(\mu)\right\}$.

Since $h$ is upper semicontinuous in $\mu$, the first integral is continuous and the second term is continuous, for each $\varepsilon>0$, there is some $\mu_{\varepsilon}$ where the supremum is attained. By compactness we can assume $\mu_{\varepsilon} \rightarrow \tilde{\mu}$. So, with the convention that $-\infty \cdot 0=0$, we find

$$
\log \lambda_{\beta} \leq \sup _{\mu \in M}\left\{\beta \int_{\pi(\omega) \in G} \log t_{\pi(\omega)} d \mu(\omega)+h(\mu)\right\} .
$$

Notice that if $\varepsilon<1$, then, according to (49),

$$
\log \lambda_{\beta, \varepsilon} \geq \sup _{\mu \in M}\left\{\beta \int_{\pi(\omega) \in G} \log t_{\pi(\omega)} d \mu(\omega)+h(\mu)\right\} .
$$

Taking limits, we obtain the reverse inequality of (50). Lemma III follows. 
LEMMA IV. There is a number $c, 0<c<1$, such that

$$
\sup _{\mu \in M}\left\{\int_{\Omega} \log t_{\pi(\omega)} d \mu(\omega)\right\} \leq \log c .
$$

Proof. Lemma IV follows from Lemma II, since if (18) holds, then if $q$ is large and $\sigma \in G(q+1)$,

$$
\frac{1}{q} \sum_{i=1}^{q} \log t_{\sigma(i), \sigma(i+1)}<\log \left(\frac{1+\Gamma}{2}\right)
$$

Thus,

$$
\int_{\Omega} \log t_{\pi(\omega)} d \mu(\omega)=\frac{1}{q}\left[q \int_{\Omega} \log t_{\pi(\omega)} d \mu(\omega)\right] .
$$

By the shift-invariance of $\mu$,

$$
\begin{aligned}
\int_{\Omega} \log t_{\pi(\omega)} d \mu(\omega) & =\frac{1}{q}\left[\sum_{i=0}^{q-1} \int_{\Omega} \log t_{\pi\left(S^{i}(\omega)\right)} d \mu(\omega)\right] \\
& =\int_{\Omega} \log \left[\prod_{i=0}^{q-1} t_{\pi\left(S^{i}(\omega)\right)}\right]^{1 / q} d \mu(\omega) \leq \log \left(\frac{1+\Gamma}{2}\right) .
\end{aligned}
$$

Proof of Theorem 2. Since $A_{\beta} \geq 0$, it follows from the Frobenius-Perron theorem that $A_{\beta}$ has a nonnegative eigenvalue $\lambda_{\beta} \geq 0$ with spectral radius $\Phi(\beta)=$ $\lambda_{\beta}$. By condition $2(\mathrm{a})$, and the fact that $\lambda_{\beta}$ is at least as large as the minimal row sum, we have $\Phi(\beta)=\lambda_{\beta}>0$ and $\Phi(0) \geq 1$. (It is possible that $\Phi(0)=1$ as Example 1 shows.)

It follows from Lemma IV that for $\beta \geq 0$ and $\varepsilon>0$

$$
\begin{aligned}
\log \Phi(\beta+\varepsilon) & =\sup _{\mu \in M}\left\{\beta \int_{\Omega} \log t_{\pi(\omega)} d \mu(\omega)+h(\mu)+\varepsilon \int_{\Omega} \log t_{\pi(\omega)} d \mu(\omega)\right\} \\
& \leq \varepsilon \log c+\log \Phi(\beta) .
\end{aligned}
$$

Thus,

$$
\Phi(\beta+\varepsilon) \leq c^{\varepsilon} \Phi(\beta) .
$$

Of course, this implies that $\Phi$ is strictly decreasing and converges to zero at infinity. This completes the proof of Theorem 2 .

To continue the analysis, let $f$ be the map of $G^{\infty}$ into $\mathbf{R}^{m}$ defined for each $\sigma \in G^{\infty}$, by

$$
\{f(\sigma)\}=\bigcap_{n=1}^{\infty} J_{\sigma \mid n} .
$$

It follows from (14) and Lemma II that $f$ is a continuous map of $G^{\infty}$ onto $K$.

DEFINITIONS. Set $\delta=\min _{(i, j) \in G}\left\{\left(t_{i, j} \operatorname{diam} J_{j}\right) / \operatorname{diam} J_{i}\right\}$ and let

$$
a=\min \left\{\lambda^{m}\left(\operatorname{int} J_{i}\right) /\left(\operatorname{diam} J_{i}\right)^{m}\right\},
$$

where $\lambda^{m}$ is Lebesgue measure on $\mathbf{R}^{m}$. 
Lemma V. Suppose $E \subset \mathbf{R}^{m}$ with $\operatorname{diam} E<\min \left\{\operatorname{diam} J_{i}\right\}$. Let

$$
B=\left\{\sigma \in G^{*} \mid \operatorname{diam} J_{\sigma}<\operatorname{diam} E \leq \operatorname{diam} J_{\sigma|| \sigma \mid-1} \text { and } E \cap J_{\sigma} \neq \varnothing\right\} .
$$

Then

$$
\# B \leq(L / a)(2 / \delta)^{m},
$$

where $L$ is the Lebesgue measure of the unit ball in $\mathbf{R}^{m}$.

ProOF. Note that if $\sigma \in G^{*}$, then

(61) $\operatorname{diam} J_{\sigma} / \operatorname{diam} J_{\sigma|| \sigma \mid-1}=\left(t_{\sigma(|\sigma|-1), \sigma(|\sigma|)} \operatorname{diam} J_{\sigma(|\sigma|)}\right) / \operatorname{diam} J_{\sigma(|\sigma|-1)} \geq \delta$.

Thus, for each $\sigma \in B$, diam $J_{\sigma} \geq \delta \operatorname{diam} E$. Since the sets $J_{\sigma}$ with $\sigma$ in $B$ are nonoverlapping and all lie in a ball, $W$, of radius $2 \operatorname{diam} E$, (62)

$$
\begin{aligned}
L(2 \operatorname{diam} E)^{m} & =\lambda^{m}(W) \geq \sum_{\sigma \in B} \lambda^{m}\left(\operatorname{int} J_{\sigma}\right) \\
& \geq \sum_{\sigma \in B}\left(\operatorname{diam} J_{\sigma}\right)^{m} \lambda^{m}\left(\operatorname{int} J_{\sigma(|\sigma|)}\right) /\left(\operatorname{diam} J_{\sigma(|\sigma|)}\right)^{m} \\
& \geq a \sum_{\sigma \in B}\left(\operatorname{diam} J_{\sigma}\right)^{m} \geq a \sum_{\sigma \in B}(\delta \operatorname{diam} E)^{m} \geq a(\operatorname{diam} E)^{m} \delta^{m} \# B .
\end{aligned}
$$

Thus,

$$
\# B \leq(L / a)(2 / \delta)^{m} \text {. }
$$

Proof of TheOREM 3. Since $A_{\alpha}$ is irreducible, by the Frobenius-Perron theorem, there is a unique strictly positive column vector

$$
v=\left[\begin{array}{c}
v_{1} \\
\vdots \\
v_{n}
\end{array}\right]
$$

with $\sum_{i=1}^{n} v_{i}=1$ and $A_{\alpha} v=v$. Thus, for each $i$,

$$
v_{i}=\sum_{j=1}^{n} t_{i j}^{\alpha} v_{j}=\sum_{(i, j) \in G} t_{i j}^{\alpha} v_{j} .
$$

Define a probability measure $\hat{\mu}$ on $G^{\infty}$ by setting for each $\sigma \in G^{*}$,

$$
\hat{\mu}([\sigma])=t_{\sigma}^{\alpha} v_{\sigma(|\sigma|)},
$$

where

$$
[\sigma]=\left\{\tau \in G^{\infty}:\left.\tau\right|_{|\sigma|}=\sigma\right\} .
$$

To see that Kolmogorov's consistency theorem may be applied it is sufficient to note that if $\sigma \in G^{*}$, then

$$
\begin{aligned}
\sum_{(\sigma(|\sigma|), j) \in G} \hat{\mu}([\sigma * j]) & =\sum t_{\sigma}^{\alpha} v_{j}=t_{\sigma}^{\alpha} \sum t_{\sigma(|\sigma|), j}^{\alpha} v_{j} \\
& =t_{\sigma}^{\alpha} v_{\sigma(|\sigma|)}=\hat{\mu}([\sigma]) .
\end{aligned}
$$


First, we will show that $\mathscr{H}^{\alpha}(K)<+\infty$. For each $p$, we have

$$
\sum_{\sigma \in G(p)}\left(\operatorname{diam} J_{\sigma}\right)^{\alpha}=\sum_{\sigma \in G(p)} t_{\sigma}^{\alpha} \operatorname{diam} J_{\sigma(|\sigma|)}^{\alpha},
$$

and since $v$ is strictly positive,

$$
\begin{aligned}
& =\sum_{\sigma \in G(p)} \hat{\mu}([\sigma])\left(\operatorname{diam} J_{\sigma(|\sigma|)}\right)^{\alpha} / v_{\sigma(|\sigma|)} \\
& \leq \sup \left\{\left(\operatorname{diam} J_{i}\right)^{\alpha} / v_{i}\right\} \sum_{\sigma \in G(p)} \hat{\mu}([\sigma]) \\
& \leq \sup \left\{\left(\operatorname{diam} J_{i}\right)^{\alpha} / v_{i}\right\}<+\infty .
\end{aligned}
$$

It follows from Lemma II that

$$
\lim _{p \rightarrow \infty} \sup \left\{\operatorname{diam} J_{\sigma} \mid \sigma \in G(p)\right\}=0 .
$$

Thus,

$$
\mathscr{H}^{\alpha}(K) \leq \sup \left\{\left(\operatorname{diam} J_{i}\right)^{\alpha} / v_{i}\right\}<+\infty .
$$

In order to show $0<\mathscr{H}^{\alpha}(K)$, transfer $\hat{\mu}$ to a probability measure on $K$. Let $\mu=\hat{\mu} \circ f^{-1}$. We will show that there is some $c>0$ such that if $E$ is a Borel subset of $\mathbf{R}^{m}$ with $\operatorname{diam} E<\inf \left\{\operatorname{diam} J_{i}\right\}$, then

$$
\mu(E) \leq c(\operatorname{diam} E)^{\alpha} .
$$

Of course, this inequality implies

$$
1 / c \leq \mathscr{H}^{\alpha}(K) .
$$

Set $B=\left\{\sigma \in G^{*} \mid \operatorname{diam} J_{\sigma}<\operatorname{diam} E \leq \operatorname{diam} J_{\sigma|| \sigma \mid-1}\right.$ and $\left.E \cap J_{\sigma} \neq \varnothing\right\}$. Note that if $\tau \in G^{*}$, and $f(\tau) \in E$, then there is some $\sigma \in B$ such that $\tau$ extends $\sigma$. Therefore,

$$
\mu(E) \leq \sum_{\sigma \in B} \hat{\mu}([\sigma]) .
$$

For any $\sigma$, we have

$$
\hat{\mu}([\sigma]) /\left(\operatorname{diam} J_{\sigma}\right)^{\alpha}=v_{\sigma(|\sigma|)} /\left(\operatorname{diam} J_{\sigma(|\sigma|)}\right)^{\alpha} .
$$

Using this equality, we find

$$
\mu(E) \leq(\operatorname{diam} E)^{\alpha} \sup \left\{v_{i} /\left(\operatorname{diam} J_{i}\right)^{\alpha}\right\} \#(B) .
$$

By Lemma $\mathrm{V}, \sup \left\{v_{i} /\left(\operatorname{diam} J_{i}\right)^{\alpha}\right\} \#(B)$ is bounded by some fixed $c>0$. Therefore, (72) holds and Theorem 3 follows.

Definitions. For $H \in \operatorname{SC}(G)$ and $\tau=\left(i_{1}, \ldots, i_{p}\right) \in G(p)$ such that $i_{p} \in H \in$ $\mathrm{SC}(G)$ and $i_{p-1} \notin H$, define

$$
K(\tau)=\{f(\omega) \mid \omega \text { extends } \tau \text { and } \forall q \geq p, \omega(q) \in H\} .
$$

Let $C=\left\{\tau \in G^{*} \mid \exists H \in \mathrm{SC}(G)\right.$ with $\tau(|\tau|) \in H$ and $\left.\tau(|\tau|-1) \notin H\right\}$.

PROOF OF THEOREM 4. It follows from the factor theorem, (6) that

$$
\alpha=\max \left\{\alpha_{H} \mid H \in \operatorname{SC}(G)\right\} .
$$


For each $\omega \in G^{\infty}$, there exist a positive integer $p(\omega)$ and $H(\omega) \in \operatorname{SC}(G)$ such that if $q \geq p(\omega)$, then $\omega(q)$ is in the vertex set of $H(\omega)$ and $\omega(p(\omega)-1)$ is not in the vertex set of $H(\omega)$. Obviously,

$$
K=\bigcup_{\tau \in C} K(\tau) .
$$

For each $\tau \in C$,

$$
\mathscr{H}^{\alpha}(K(\tau))=t_{\tau}^{\alpha} \mathscr{H}^{\alpha}\left(K_{H}(\tau(|\tau|))\right)<+\infty .
$$

Thus, $K$ has $\sigma$-finite $\mathscr{H}^{\alpha}$ measure. From (78) and Theorem $3, \mathscr{H}^{\alpha}(K)>0$.

Case 1. Suppose $M=\left\{H \mid \alpha_{H}=\alpha\right\}$ consists of incomparable elements. Since $\mathscr{H}^{\alpha}(K(\tau))=0$, unless $\tau(|\tau|) \in H \in M$, we have

$$
\begin{aligned}
\mathscr{H}^{\alpha}(K) & \leq \sum_{H \in M} \sum_{\substack{\tau \in C \\
\tau(|\tau|) \in H}} \mathscr{H}^{\alpha}(K(\tau)) \\
& \leq \sum_{H \in M} \sum_{j \in H}\left[\sum_{\substack{\tau \in C \\
\tau(|\tau|)=j}} \mathscr{H}^{\alpha}(K(\tau))\right] \\
& \leq \sum_{H \in M} \sum_{j \in H} \mathscr{H}^{\alpha}\left(K_{H}(j)\right)\left[\sum_{\substack{\tau \in C \\
\tau(|\tau|)=j}} t_{\tau}^{\alpha}\right] .
\end{aligned}
$$

So, $\mathscr{H}^{\alpha}(K)$ is finite provided this last inner sum is finite.

If $\tau \in C$ and $\tau(|\tau|)=j$, then $\tau$ travels through some transient points and possibly through points of some of the strongly connected components $H_{0}$ with $\alpha_{H_{0}}<\alpha$. Thus,

$$
\sum_{\substack{\tau \in C \\ \tau(|\tau|)=j}} t_{\tau}^{\alpha} \leq\left[\prod_{\substack{i \in \operatorname{Trans} \\(i, k) \in G}}\left(1+t_{i, k}^{\alpha}\right)\right]\left[\prod_{\substack{i \in \operatorname{Trans} \\(k, i) \in G}}\left(1+t_{k, i}^{\alpha}\right)\right] \prod_{\substack{H_{0} \in \operatorname{SC}(G) \\ \alpha_{H_{0}} \neq \alpha}}\left[1+\sum_{\mathfrak{z} \in H_{0}^{*}} t_{\mathfrak{z}}^{\alpha}\right] .
$$

The first two factors are fixed finite numbers. To see that the third factor is finite, it suffices to fix $H_{0}$ with $\alpha_{H_{0}}<\alpha$ and note

$$
\sum_{\mathfrak{z} \in H_{0}^{*}} t_{\mathfrak{z}}^{\alpha}=\sum_{n=2}^{\infty} \sum_{|\mathfrak{z}|=n} t_{\mathfrak{z}}^{\alpha} .
$$

We apply the root test to this last series:

$$
\left[\sum_{|z|=n} t_{z}^{\alpha}\right]^{1 / n} \leq\left[\max _{\substack{z \in H_{0}^{*} \\|z|=n}} t_{z}^{\alpha-\alpha\left(H_{0}\right)}\right]^{1 / n} \times\left[\sum_{\substack{z \in H_{0}^{*} \\|z|=n}} t_{z}^{\alpha\left(H_{0}\right)}\right]^{1 / n} .
$$


Now, since the construction restricted to $H_{0}$ is still a construction, Lemma II is applicable and

$$
\varlimsup_{n \rightarrow \infty}\left[\sum_{|z|=n} t_{z}^{\alpha}\right]^{1 / n} \leq \Gamma_{H_{0}}^{\alpha-\alpha\left(H_{0}\right)} \varlimsup_{n \rightarrow \infty}\left[\sum_{\substack{z \in H_{0}^{*} \\|z|=n}} t_{\mathfrak{z}}^{\alpha\left(H_{0}\right)}\right]^{1 / n} .
$$

Since $H_{0}$ is irreducible, the proof of Theorem III shows that the sums $\sum_{\mathfrak{z} \in H_{0}^{*}} t_{\mathfrak{z}}^{\alpha\left(H_{0}\right)}$ are uniformly bounded. The root test now yields the convergence of the series.

Case 2. In this case, there exist distinct elements $H_{1}$ and $H_{2}$ in $M$ and a path $\gamma=\left(g_{1}, \ldots, g_{k}\right)$ such that $g_{1} \in H_{1}, g_{k} \in H_{2}$ and $g_{i} \notin H_{1} \cup H_{2}$, if $1<i<k$. Let us assume that $H_{1}=\left\{1, \ldots, m_{1}\right\}, H_{2}=\left\{m_{1}+1, \ldots, m_{1}+m_{2}\right\}, g_{1}=1$ and $g_{k}=m_{1}+1$.

For each $q$, set

$$
E_{q}=\left\{\tau \in G(q) \mid \tau \subseteq H_{1} \text { and } \tau(q)=1\right\},
$$

$$
F_{q}^{*}=\left\{\eta \in G^{*} \mid \eta=\tau * \tilde{\gamma} * \sigma, \text { where } \tau \in E_{q}, \sigma \in H_{2}^{*}, \sigma(1)=m_{1}+1\right\},
$$

and

$$
F_{q}=\left\{\eta \in G^{\infty} \mid \eta=\tau * \tilde{\gamma} * \sigma, \text { where } \tau \in E_{q}, \sigma \in H_{2}^{\infty}, \sigma(1)=m_{1}+1\right\},
$$
where $\tilde{\gamma}=\left(g_{2}, \ldots, g_{k-1}\right)$. Let

$$
v_{H_{2}}=\left[\begin{array}{c}
v_{m_{1}+1} \\
\vdots \\
v_{m_{1}+m_{2}}
\end{array}\right]
$$

be the strictly positive column eigenvector of $A_{H_{2}, \alpha}$ with $\sum_{i=1}^{m_{2}} v_{m_{1}+i}=1$. Define $\hat{\mu}_{q}$ on $F_{q}$ by

$$
\hat{\mu}_{q}([\tau * \tilde{\gamma} * \sigma])=\left(t_{\tau}^{\alpha} t_{\gamma}^{\alpha} t_{\sigma}^{\alpha}\right) v_{\sigma(|\sigma|)},
$$

where $\sigma \in H_{2}^{*}$ and $\sigma(1)=m_{1}+1$. As before, transfer $\hat{\mu}_{q}$ to $\mu_{q}$, a measure on $K(1)$ and, therefore, on $K$. For each $r$, set

$$
\nu_{r}=\sum_{q=1}^{r} \mu_{q} .
$$

The fact that $K$ does not have finite $\mathscr{H}^{\alpha}$ measure follows from the next two claims:

Claim 1. There is some $c, 0<c<+\infty$ such that for each $r$, there exists some $b_{r}>0$ such that

$$
\nu_{r}(E) \leq c(\operatorname{diam} E)^{\alpha},
$$

provided $\operatorname{diam} E<b_{r}$.

ProOF OF ClaIM 1. Let $b_{r}=\inf \left\{\operatorname{diam} J_{\sigma} \mid \sigma=\tau * \tilde{\gamma} * m_{1}+1\right.$, where $\tau \in$ $\left.\bigcup_{q \leq r} E_{q}\right\}$ and let $\operatorname{diam} E<b_{r}$. Let

(93) $B=\left\{\eta \in \bigcup_{q \leq r} F_{q}^{*} \mid \operatorname{diam}\left(J_{\eta}\right)<\operatorname{diam} E \leq \operatorname{diam} J_{\eta|| \eta \mid-1}\right.$ and $\left.E \cap J_{\eta} \neq \varnothing\right\}$, 
and for each $q$, let

$$
B_{q}=\left\{\eta \in B \mid \eta \in F_{q}^{*}\right\} .
$$

The sets $B_{q}$ are pairwise disjoint and

$$
\begin{aligned}
\nu_{r}(E) & =\sum_{q \leq r} \mu_{q}(E) \leq \sum_{q \leq r} \sum_{\eta \in B_{q}} \hat{\mu}_{q}([\eta]) \\
& \leq \sum_{q \leq r} \# B_{q}(\operatorname{diam} E)^{\alpha} \sup \left\{v_{i} /\left(\operatorname{diam} J_{i}\right)^{\alpha} \mid m_{1}+1 \leq i \leq m_{1}+m_{2}\right\} \\
& \leq \# B(\operatorname{diam} E)^{\alpha} .
\end{aligned}
$$

Set $S$ equal to the last supremum. Using Lemma V, we have

$$
\nu_{r}(E) \leq S(L / a)(2 / \delta)^{m}(\operatorname{diam} E)^{\alpha} .
$$

Since $S, L, a$, and $\delta$ are independent of $r$ the claim follows.

Now, Claim 1 implies

$$
\mathscr{H}^{\alpha}(K) \geq \lim _{r \rightarrow \infty}\left(\frac{1}{c}\right) \nu_{r}(K) \geq\left(\frac{1}{c}\right) \sum_{q=1}^{\infty} \hat{\mu}_{q}\left(F_{q}\right)=\left(\frac{1}{c}\right) t_{\gamma}^{\alpha} \sum_{q=1}^{\infty} \sum_{\tau \in E_{q}} t_{\tau}^{\alpha} .
$$

The proof of the theorem follows from the next claim.

Claim 2. $\sum_{q=1}^{\infty} \sum_{\tau \in E_{q}} t_{\tau}^{\alpha}=\infty$.

By the Frobenius-Perron theorem, there is a unique strictly positive row vector $u=\left(u_{1}, \ldots, u_{m_{1}}\right)$ with $\sum u_{i}=1$ such that

$$
u A_{H_{1}, \alpha}=u \text {. }
$$

It also follows that

$$
\frac{1}{n} \sum_{q=1}^{n}\left\langle e_{1},(1, \ldots, 1) A_{H_{1}, \alpha}^{q}\right\rangle
$$

converges to $u_{1}>0$, where $e_{1}$ is the unit vector $(1,0, \ldots, 0)$ in $\mathbf{R}^{m_{1}}$. But,

$$
\sum_{\tau \in E_{q}} t_{\tau}^{\alpha}=\left\langle e_{1},(1, \ldots, 1) A_{H_{1}, \alpha}^{q}\right\rangle .
$$

This means the series diverges and Theorem 4 follows.

We can now analyse each $K_{j}$. To do this, let $C_{j}=\{H \in \mathrm{SC}(G) \mid$ there is a path from $j$ to some vertex of $H$ \}.

THEOREM 5. $\operatorname{dim} K_{j}=\alpha_{j}=\max \left\{\alpha_{H} \mid H \in C_{j}\right\}$ and $K_{j}$ has positive, $\sigma$-finite $\mathscr{H}^{\alpha_{j}}$ measure. Moreover, $\mathscr{H}^{\alpha_{j}}\left(K_{j}\right)<\infty$ if and only if $M_{j}=\left\{H \in C_{j} \mid \alpha_{H}=\alpha_{j}\right\}$ consists of incomparable elements.

Theorem 5 is proved by defining $G^{\prime}$ to be the restriction of $G$ to all vertices $i$ such that there is a path from $j$ to $i$ and noting that $K_{j}$ only depends on this part of the graph $G$.

EXAMPLE 1. The simplest way to obtain a construction with $\Phi(0)=1$ is to loop something on itself. Thus, $G$ has one vertex 1 and $T_{1,1}$ is a similarity contracting $J_{1}$ into itself. Of course, $K$ is a singleton. There are more interesting possibilities here. For example, take two sets $J_{1}$ and $J_{2}$, loop $J_{1}$ on itself, loop $J_{2}$ on itself and 
map $J_{1}$ into $J_{2}$. Again, $\Phi(0)=1$, but now $K$ has exactly one limit point. One can continue this procedure to generate sets $K$ with Cantor-Bendixson order $n$, for each integer $n$. With a generalization of directed constructions to infinite graphs $G$, one can build countable compact sets with derived set order $\alpha$, for any given $\alpha<\omega_{1}$.

EXAMPLE 2. Let $T_{b}(c, r)$ consist of those $x$ 's in $[0,1]$ for which any $r$ consecutive base $b$ digits in the $b$-ary expansion of $x$ sum up to at least $c$. We will derive the dimension $\alpha$ of each set $T_{b}(c, r)$ from our results. The dimension of these sets has also been given by Drobot and Turner [7]. Our results show, in addition that $0<\mathscr{H}^{\alpha}\left(T_{b}(c, r)\right)<\infty$.

For each $\left(e_{1}, \ldots, e_{r}\right) \in\{0, \ldots, b-1\}^{r}$, let

$$
\left\langle e_{1}, \ldots, e_{r}\right\rangle=\left\{x \in[0,1] \mid \text { the } b \text {-expansion of } x \text { begins with } e_{1}, \ldots, e_{r}\right\} .
$$

Of course, there are countably many $x$ 's which have more than one expansion. We can safely ignore these. Let $J_{1}, \ldots, J_{n}$ enumerate the sets $\left\langle e_{1}, \ldots, e_{r}\right\rangle$ such that

$$
\sum_{i=1}^{r} e_{i} \geq c .
$$

The graph $G$ is determined as follows. Suppose $J_{i}=\left\langle e_{1}, \ldots, e_{r}\right\rangle$ and $J_{j}=\left\langle e_{1}^{\prime}, \ldots, e_{r}^{\prime}\right\rangle$. Then $(i, j) \in G$ if and only if $J_{j}$ begins with the shift of $J_{i}$. That is,

$$
\left\langle e_{2}, \ldots, e_{r}\right\rangle=\left\langle e_{1}^{\prime}, \ldots, e_{r-1}^{\prime}\right\rangle .
$$

If $(i, j) \in G$, then $T_{i, j}$ is the natural linear map which takes $J_{j}$ onto $\left\langle e_{1}, \ldots, e_{r}, e_{r}^{\prime}\right\rangle$. Thus, $T_{i, j}$ has similarity ratio $1 / b$. Clearly, the graph $G$ itself is strongly connected. So, $0<\mathscr{H}^{\alpha}\left(T_{b}(c, b)\right)<\infty$, where $\alpha$ is the dimension of $T_{b}(c, b)$. Notice that if $M$ is the incidence matrix of $G$, then

$$
A_{\beta}=(1 / b)^{\beta} M .
$$

Thus, $\operatorname{det}\left(A_{\beta}-I\right)=(1 / b)^{\beta n} \operatorname{det}\left(M-b^{\beta} I\right)$. Therefore, $b^{\alpha}=\rho(M)$, the spectral radius of $M$. Or,

$$
\alpha=\log (\rho(M)) / \log b .
$$

EXAMPLE 3. Consider the construction given by $J_{1}=[0,1 / 3], J_{2}=[1 / 3,4 / 9]$, $J_{3}=[5 / 9,2 / 3]$ and $J_{4}=[2 / 3,1]$. The similarity maps are the orientation-preserving maps such that $T_{1}$ maps $[0,1]$ onto $J_{1}, T_{2}$ maps $[1 / 3,2 / 3]$ onto $J_{2}, T_{3}$ maps $[1 / 3,2 / 3]$ onto $J_{3}$ and $T_{4}$ maps $[0,1]$ onto $J_{4}$. Thus, $t_{1}=t_{2}=t_{3}=t_{4}=1 / 3$. The directed graph $G$ is given by the diagram:

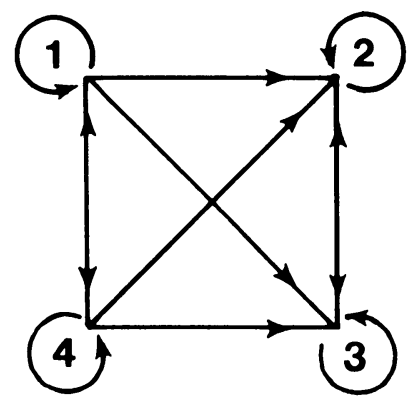

FIGURE 1 
If $(i, j) \in G$, then $T_{i, j}=T_{i}$. The strongly connected components of $G$ are $H_{1}=$ $\{1,4\}$ and $H_{2}=\{2,3\}$. Clearly,

$$
\alpha_{H_{1}}=\alpha_{H_{2}}=\alpha=\ln 2 / \ln 3 .
$$

Since there is a path from $H_{1}$ to $H_{2}$, Theorem 4 applies and we know that $K$ has infinite, but $\sigma$-finite measure. It is rather easy to see what $K$ is in this construction. One simply takes the standard middle-third Cantor set and in each complementary interval attach a similar copy of the middle-third set. Thus, on level $n$ we will have $2^{n}$ copies of the middle third set. So,

$$
\mathscr{H}^{\alpha}(K)=\sum_{n=0}^{\infty} 2^{n}\left[(1 / 3)^{n+1}\right]^{\alpha}=\sum_{n=0}^{\infty}\left(2 / 3^{\alpha}\right)^{n}\left(1 / 3^{\alpha}\right)=\infty .
$$

A ratio graph directed construction consists of a directed graph $G$ with vertex set consisting of the integers $1, \ldots, n$ and positive numbers $t_{i, j}$, where $(i, j) \in G$ such that

(a) for each $i, 1 \leq i \leq n$, there is some $j$ such that $(i, j) \in G$, and

(b) if the path component of $G$ rooted at the vertex $i_{1}$ is a cycle:

$$
\left[i_{1}, \ldots, i_{q}, i_{q+1}=i_{1}\right]
$$

then

$$
\prod_{k=1}^{q} t_{i_{k}, i_{k+1}}<1
$$

A realization of a ratio directed construction consists of a sequence of nonoverlapping compact subsets of $\mathbf{R}^{m}: J_{1}, \ldots, J_{n}$, such that each $J_{i}$ has a nonempty interior and a family of similarity maps $T_{i_{1}, \ldots, i_{k}}$ where $\left(i_{1}, \ldots, i_{k}\right) \in G^{k}$, for $k=2,3,4, \ldots$ such that the similarity ratio of $T_{i_{1}, \ldots, i_{k}}$ is given by

$$
\prod_{q=1}^{k-1} t_{i_{q}, i_{q+1}}
$$

and such that the family of sets $J_{\sigma}$, where $\sigma \in G$ forms a nonoverlapping Cantor scheme. Here, if $\sigma=\left(i_{1}, \ldots, i_{k}\right) \in G^{*}$, with $k \geq 2$, then $J_{\sigma}=T_{\sigma}\left(J_{\sigma(k)}\right)$.

The object constructed by a realization of a ratio directed construction is

$$
K=\bigcap_{p=1}^{\infty}\left[\bigcup_{\sigma \in G(p)} J_{\sigma}\right] .
$$

A geometric graph directed construction is a particular type of realization of a ratio directed construction.

Notice that all the proofs given in the paper depend only on the ratios except for the proof of Lemma I. This lemma will hold once a realization has been given. Thus, the dimension and measure of the set $K$ can be calculated exactly as before. This result generalizes Moran's ratio theorem. Again, one should note that this theorem depends only on the ratios employed.

EXAMPLE 4. This example is due to J. Marion [9]. Let $E_{1}, \ldots, E_{n}$ be compact subsets of $\mathbf{R}^{m}$ having the property that there is some $\xi>0$ such that for 
$j=1, \ldots, n, E_{j}$ is the disjoint union of $a_{1 j}$ subsets similar to $E_{1}$ each with reduction ratio $\xi$, of $a_{2 j}$ subsets similar to $E_{2}$ each with reduction ratio $\xi$, etc. Thus, in particular $E_{j}$ is the union of $\sum_{i=1}^{n} a_{i j}$ pairwise disjoint sets. Let $A$ be the integer valued matrix $\left\{a_{i j}\right\}$. Assuming that $A$ is primitive, Marion shows that for each $k=1, \ldots, n$

$$
0<\mathscr{H}^{\alpha}\left(E_{k}\right)<\infty
$$

where $\alpha=-\log ($ spectral radius $A) / \log \xi$. This result follows from Theorem 3 of this paper and we assume only that $A$ is irreducible. In order to see this, note that we can assume that the sets $E_{1}, \ldots, E_{n}$ are disjoint. Let

$$
D=\left\{(l, i, j) \mid 1 \leq l \leq a_{i j}\right\} .
$$

By Marion's hypothesis, there are disjoint sets

$$
\left\{E_{(l, i, j)} \mid(l, i, j) \in D\right\}
$$

such that for each $j, j=1, \ldots, n$,

$$
E_{j}=\bigcup_{(l, i)} E(l, i, j)
$$

and $E_{l, i, j}$ is similar to $E_{i}$ with ratio $\xi$. The directed graph for the construction has $D$ as its vertex set. There is an edge from $(l, i, j)$ to $(r, s, t)$ provided that $i=t$. The similarity map indexed by this edge is the one determined by Marion's hypothesis. It has reduction ratio $\xi$. Since the sets $E_{l, i, j}$ are disjoint, there is some $\delta>0$ such that if each $E_{l, i, j}$ is replaced by $J_{l, i, j}$, its closed $\delta$ neighbourhood, they are still disjoint. We now have a graph directed construction with incidence matrix $A_{0}$. A direct application of Theorem 4 shows that $0<\mathscr{H}^{\alpha}\left(E_{k}\right)$ and the $\mathscr{H}^{\alpha}$ measure of $E_{k}$ is $\sigma$-finite, where $\alpha=-\log \left(\right.$ spectral radius $\left.A_{0}\right) / \log \xi$. To obtain Marion's result we first check that

$$
\text { spectral radius of } A_{0}=\text { spectral radius of } A \text {. }
$$

To see this consider a column vector $v=\left\{v_{(r, s, t)} \mid(r, s, t) \in D\right\}$ such that $A_{0} v=\lambda v$. So,

$$
\lambda v_{(l, i, j)}=\sum_{(r, s, t)} a_{((l, i, j),(r, s, t))} v_{(r, s, t)} .
$$

Since $a_{((l, i, j),(r, s, t))}=0$ unless $i=t$, and is one if $i=t$,

$$
\lambda v_{(l, i, j)}=\sum_{\substack{(r, s) \\(r, s, i) \in D}} v_{(r, s, i)} .
$$

This sum depends only on $i$. So, there are numbers $w_{1}, \ldots, w_{n}$ such that $v_{l, i, j}=w_{i}$. Thus,

$$
\begin{aligned}
\lambda w_{i} & =\sum_{\substack{(r, s) \\
(r, s, i) \in D}} w_{s} \\
& =\sum_{s=1}^{n} \sum_{1 \leq r \leq a_{s, i}} w_{s} \\
& =\sum_{s=1}^{n} a_{s, i} w_{s} .
\end{aligned}
$$


Or, $\lambda w=A^{t} w$. It follows from this that the spectral radius of $A$ is the spectral radius of $A_{0}$.

Finally, we note that with some effort it can be shown that $A$ is irreducible if and only if $A_{0}$ is irreducible.

EXAMPLE 5. This example is due to H. Cajar [10]. It generalizes the example of Drobot and Turner and is related to similar results concerning sets determined by their arithmetic expansions. These results are referenced in Cajar's book. Let $g$ be an integer, $g \geq 2$ and $C=\{0,1, \ldots, g-1\}$. Let $l$ be an integer greater than one and $B \subset C^{l}$. We will consider the $g$-ary expansion of numbers $x$ in $[0,1]$. For our considerations we can neglect countable sets. If $\sum_{i=1}^{\infty} x_{i} / g^{i}$, with each $x_{i} \in C$, then $b_{i}^{l}(x):=\left(x_{i}, x_{i+1}, \ldots, x_{i+l-1}\right)$. Set $X_{B}=\left\{x \mid \forall i b_{i}^{l}(x) \in B\right\}$. Now, $X_{B}$ can be determined as a graph directed construction as follows. First, build a graph on $B$ by having a directed edge from $b_{1}=\left(b_{1}^{1}, \ldots, b_{l}^{1}\right)$ to $\left(b_{1}^{2}, \ldots, b_{l}^{2}\right)$ provided $\left(b_{2}^{1}, b_{3}^{1}, \ldots, b_{l}^{1}\right)=\left(b_{1}^{2}, \ldots, b_{l-1}^{2}\right)$. This directed graph may not be the graph of a construction since there may be some vertex with no arrow coming out of it. So, lop off all such vertices and edges into such vertices. We obtain a derived graph. Iterate this procedure. Evidently, we will obtain the empty set or else we will obtain a directed graph which satisfies condition $2(\mathrm{a})$ and is a construction. In the first case, $X_{B}=\varnothing$. In the second case, we obtain a subset $D \subset A^{l}$ such that $X_{B}=X_{D}$. The graph on $D$ obviously directs a construction of $X_{B}$. The construction matrix $A$ is the function: $A\left(b_{1}, b_{2}\right)=1 / g^{l}$ if $b_{1}, b_{2} \in D$ and $=0$ otherwise. Thus, $\operatorname{dim}_{H}\left(X_{B}\right)=\alpha$, where the spectral radius of $A_{\alpha}=1$. But, $A_{\alpha}=(1 / g)^{\alpha} A_{0}$, where $A_{0}$ is the incidence matrix of $D$. So, we have the equation $1=g^{-\alpha}$ (spectral radius $\left.A_{0}\right)$. Thus,

$$
\operatorname{dim}_{H}\left(X_{B}\right)=\log \left(\text { spectral radius } A_{0}\right) / \log g \text {. }
$$

It is easy to see that if $M$ is the incidence matrix of $G$, then $M$ and $A_{0}$ have the same spectral radius. Thus,

$$
\operatorname{dim}_{H}\left(X_{B}\right)=\log (\operatorname{spectral} \text { radius } M) / \log g .
$$

This is Cajar's formula. From the results of this paper, we know, in addition that the $\mathscr{H}^{\alpha}$ measure of $X_{B}$ is positive and either finite or $\sigma$-finite. One can construct examples to show that both cases are possible.

\section{REFERENCES}

1. P. A. P. Moran, Additive functions of intervals and Hausdorff measure, Proc. Cambridge Philos. Soc. 42 (1946), 15-23.

2. J. E. Hutchinson, Fractals and self-similarity, Indiana Univ. Math. J. 30 (1981), 713-747.

3. R. S. Ellis, Entropy, large deviations, and statistical mechanics, Springer-Verlag, New York, 1985.

4. Peter Walters, An introduction to ergodic theory, Springer-Verlag, New York, 1982.

5. P. Billingsley, Ergodic theory and information, Wiley, New York, 1965.

6. K. J. Falconer, The geometry of fractal sets, Cambridge Univ. Press, New York, 1985.

7. V. Drobot and J. Turner, Hausdorff dimension and Perron-Frobenius theory, Illinois J. Math. 32 (1988).

8. C. A. Rogers, Hausdorff measures, Cambridge Univ. Press, New York, 1970.

9. J. Marion, Mesures de Hausdorff et théorie de Perron-Frobenius des matrices non-negatives, Ann. Inst. Fourier (Grenoble) 35 (1985), 99-125. 
10. H. Cajar, Billingsley dimension in probability spaces, Lecture Notes in Math., vol. 892, SpringerVerlag, New York, 1981.

11. T. Bedford, Dynamics and dimension for fractal recurrent sets, J. London Math. Soc. (2) 33 (1986), 89-100.

Department of Mathematics, University of North Texas, Denton, Texas 76203-5116

Department of Mathematics, Utah State University, logan, Utah 84322 